\title{
Acquisition of behavioral control by the auditory features of an imprinting object*
}

\author{
LEONARD A. EISERER and HOWARD S. HOFFMAN \\ Bryn Mawr College, Bryn Mawr, Pennsylvania 19010
}

\begin{abstract}
Presentations of a visible moving object immediately suppressed ongoing distress vocalization in newly hatched ducklings, but presentations of only the auditory features of the object initially had no effect. However, as the ducklings received more and more exposure to the visible moving object, the object's auditory features gradually acquired the ability to suppress distress calls themselves. These auditory features failed to develop suppressive properties in ducklings which received prolonged exposure to those features but never saw the object in motion. The gradual acquisition of behavioral control by the auditory features of a moving object is taken to represent the essence of the imprinting process.
\end{abstract}

When an arbitrarily selected moving object is initially presented to newly hatched ducklings that are emitting distress calls, those calls are almost totally suppressed (Hoffman, Stratton, Newby, \& Barrett, 1970). This suppression, which is generally considered a basic component of the filial response (Sluckin, 1965), is so immediate as to imply that it represents an unlearned reaction to some aspect of the stimulation provided by the object. Based on a number of findings, it is now apparent that the stimulation provided by the motion of the object is the critical factor. Thus suppression does not occur when newly hatched ducklings are first exposed to sounds of the object but do not also see it move, nor does it occur if the object remains stationary and silent during its initial presentation (Hoffman et al, 1970).

In short, with the kinds of arbitrarily selected imprinting stimuli used in most experiments with ducklings, the static visual features of the imprinting object (as represented by the object remaining stationary), as well as its specific auditory features (i.e., the sound of the moving but visually hidden object), are functionally neutral in their initial effects upon ongoing distress vocalization. This, of course, does not preclude the possibility that, for other species of precocial birds (e.g., chicks), mere auditory stimulation might be sufficient to elicit filial responses. Moreover, it is possible that with ducklings, in a natural setting and with a natural mother, other sorts of stimulation besides visual motion (e.g., the mother's specific calls, her specific tactile stimulation, or some of her distinctive visual features) might also innately elicit filial behavior. But in the laboratory setting, these species-specific signals are not ordinarily components of the arbitrarily selected imprinting objects that are typically used (some laboratory workers do employ "natural" imprinting objects, but these are in the minority).

*This research was supported by Grant MH 19715 from the National Institute of Mental Health. Requests for reprints should be sent to Howard S. Hoffman, Department of Psychology, Bryn Mawr College, Bryn Mawr, Pennsylvania 19010.
Although the static visual features of a laboratory imprinting object may not initially elicit filial behavior, these features can gradually acquire strong suppressive properties if the ducklings receive prolonged visual exposure to the object in motion (Hoffman, Eiserer, \& Singer, 1972). Thus Hoffman et al found that in ducklings which periodically saw the imprinting object in motion, the static visual features (i.e., the object when it remained stationary) by themselves gradually acquired the ability to suppress distress calls. Prolonged exposure to only the static visual features, however, was not sufficient for the acquisition of suppressive properties-that is, repeated exposure to the object in motion was needed.

In the research reported here, we examined the development of behavioral control by the auditory features of an imprinting object in ducklings which occasionally saw the object in motion, as well as in ducklings which heard the object while it was moving but never saw it. Several investigators have reported that various auditory stimuli can themselves control following and choice behavior if they have been associated with a visible moving object (Klopfer, 1959; Gottlieb, 1965; Fischer, 1966), but those studies did not track the development of this control.

Specification of the course of this development is important because of its relevance to the theoretical interpretation of imprinting. Hoffman and Ratner (1973), for example, have hypothesized that "imprinting is a process of primary reinforcement in which the distinctive (and initially neutral) features of a given stimulus gradually acquire the capacity to elicit filial behavior [p. 531]." According to this view, the gradual development of suppressive properties by these features results from their association with the stimulation provided by visual motion, and, as such, this development reflects the essence of the imprinting process itself.

As we have already noted, Hoffman et al tracked the development of control by the static visual features of an imprinting object. By doing so, they provided what 


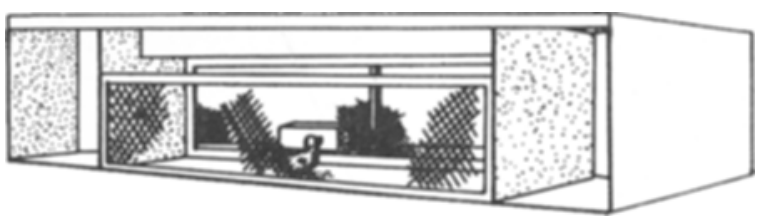

Fig. 1. The imprinting apparatus.

appeared to be a direct view of the imprinting process. In the present study, we sought to track the acquisition of control by the auditory features of an imprinting object, and in this fashion, we hoped to provide another opportunity to view the imprinting process directly.

\section{METHOD}

\section{Subjects}

The present study employed eight Khaki Campbell ducklings, Anas platyrhynchos domesticus, that were hatched in visual isolation from eggs obtained from George F. Shaw, Inc., West Chester, Pennsylvania. Except for periods spent in the experimental apparatus, each duckling was maintained in an individual housing unit consisting of a 15 -gal white translucent container that was lined with a clear polyethylene bag and was partially filled with bedding material. Under these circumstances, the ducklings could hear each other but their visual environment was restricted to that provided by the inside of their individual housing units. While in these units, the ducklings had continuous access to food and water. The room in which the housing units were maintained, as well as the room in which the ducklings were subsequently tested, were kept at a temperature of approximately $28^{\circ} \mathrm{C}$.

\section{Apparatus}

The experimental apparatus is illustrated in Fig. 1. It consisted of a plywood box $(122 \times 76 \times 76 \mathrm{~cm})$ divided lengthwise by a fine-mesh stainless steel screen, into approximately equal compartments, one for the duckling and the other for the imprinting object. Lighting in the $S$ compartment was provided by two continuously illuminated $75-\mathrm{W}$ incandescent lamps mounted above the screen. These lamps were positioned so that, unless the stimulus compartment was also illuminated, the light which reflected from the stainless steel screen prevented the $S$ from seeing into the stimulus compartment.

The imprinting object consisted of a white rectangular foam-rubber covering $(22 \times 10 \times 10 \mathrm{~cm})$ mounted over a model train engine which ran the length of the stimulus compartment on HO-ga track. Presentations of the visible moving object were produced by illuminating two overhead $75-\mathrm{W}$ incandescent lamps in.its compartment and moving it back and forth along its track at approximately $30 \mathrm{~cm} / \mathrm{sec}$. Presentations of only the auditory features of the imprinting object were produced by moving the object back and forth along its track without illuminating the lamps in the stimulus compartment. The sound thus produced was a broad-band, low-frequency noise that moved within the stimulus compartment; its average intensity was $67 \mathrm{~dB}$ re $0.0002 \mathrm{dyne} / \mathrm{cm}^{2}$. Complete stimulus withdrawal was accomplished by extinguishing the compartment lamps and stopping the movement of the object.

Distress vocalization of the ducklings was monitored with a specially constructed voice key which, through selective filtering, was sensitive only to those sounds which fell in the frequency range characteristic of distress calls (approximately $3,000-4,000 \mathrm{~Hz}$ ).

\section{Procedure}

Upon hatching, the ducklings were randomly divided into two groups, each containing four birds. Each group received a total of five experimental sessions, at the rate of 1 session/day, beginning approximately $12 \mathrm{~h}$ posthatch.

For Ss in Group 1, each experimental session consisted of a 20-min exposure to the moving object, immediately followed by a brief distress vocalization test. During this test, the auditory features and the moving object were each presented, in random sequence, for three $30-\mathrm{sec}$ periods. Each of these stimulus presentations was immediately followed by $30 \mathrm{sec}$ of complete stimulus withdrawal before the next presentation occurred. After the test was completed, the duckling was returned to its housing unit.

For birds in Group 2, each session consisted of a 20-min exposure to only the auditory features, followed by three 30 -sec presentations and withdrawals of those features.

\section{RESULTS}

For a given duckling the suppression of distress calls by the visible moving object was assessed by calculating the ratio: $(B-M) /(B+M)$, where B represents seconds of distress calling per minute which occurred during the several baseline intervals (i.e., during complete stimulus withdrawal), and $M$ represents seconds of distress calling per minute emitted during presentations of the moving object. With this index, a ratio of +1.0 indicates perfect suppression by the stimulus, while ratios which approximate zero indicate that distress vocalization in the presence of the stimulus was essentially the same as distress vocalization in its absence.

To evaluate the effects of the auditory features on distress vocalization, a similar ratio was used: $(B-A) /(B+A)$, where $A$ represents seconds of distress calling per minute during presentations of the auditory features, and B again represents seconds of distress calling per minute during the baseline intervals.

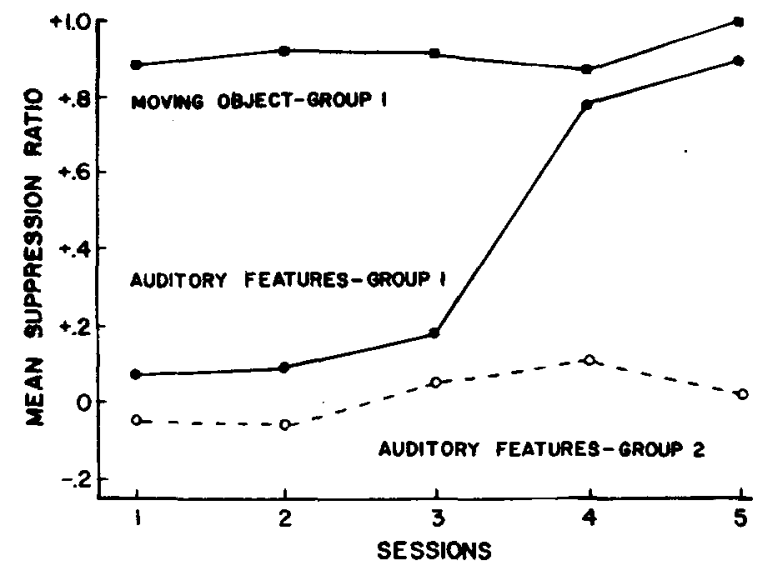

Fig. 2. Acquisition of control over distress vocalization by the auditory features of the imprinting object. Ducklings in Group 1 were exposed to the visible moving object. Ducklings in Group 2 were never exposed to the visible moving object but only to the auditory features alone. 
Although baseline rates of distress vocalization varied somewhat from bird to bird and from session to session, most birds vocalized for $13-29 \mathrm{sec} / \mathrm{min}$ of baseline $(\bar{X}=18.9 \mathrm{sec}$ for Group 1, $20.7 \mathrm{sec}$ for Group 2). An analysis of variance for repeated measures failed to detect either a reliable difference in the rates of baseline distress vocalization of the two groups $(\mathrm{F}=1.2, \mathrm{df}=1,6$, $\mathrm{p}>.05)$ or a reliable change in baseline rates across session $(F=2.4, d f=4,24, p>.05)$.

Figure 2 depicts the average suppression ratios produced by presentations of the visible moving object and of the auditory features to ducklings in Group 1, and by presentations of the auditory features to ducklings in Group 2. As the figure makes clear, the auditory features never developed suppressive control in ducklings which did not see the moving object. In contrast, the auditory features did gradually acquire suppressive properties for ducklings that received lengthy exposure to the moving object, which had strong suppressive properties from the beginning.

Repeated measure analyses of variance tests were employed to evaluate the statistical reliability of these findings. An analysis of variance for the suppression ratios generated by presentations of the moving object and of the auditory features in Group 1 revealed a significant stimulus effect $(F=43.8, d f=1,3 p<.05)$, a significant sessions effect $(F=28.6, d f=4,12, p<.05)$, and a significant interaction $(F=21.9, \quad d f=4,12$, $p<.05$ ). Comparison of the suppression afforded by the auditory features in Groups 1 and 2 revealed a significant group difference $(F=36.3, d f=1,6, p<.05)$, a significant sessions effect $(\mathrm{F}=37.0, \mathrm{df}=4,24$, $\mathrm{p}<.05)$, and a significant interaction $(\mathrm{F}=25.0$, $\mathrm{df}=4,24, \mathrm{p}<.05$ ). In summary, all of the major effects depicted in Fig. 2 are statistically reliable.

\section{DISCUSSION}

For ducklings in Group 1, suppression of distress vocalization by the visible moving object was powerful upon the earliest exposures. This finding is consistent with the results of previous work in our laboratory (Hoffman et al, 1970; Hoffman et al, 1972) and, as already noted, suggests the operation of an unlearned response.

In contrast to immediate suppression by the visible moving object, the auditory features alone-like the static visual features-initially had little effect upon ongoing distress vocalization. The auditory features appeared to be further comparable to the static visual features in that both kinds of stimulation gradually acquired suppressive control if, and only if, the moving object was sometimes seen. Of course, the functional properties of a given auditory stimulus probably vary among bird species and among differences in the nature of the stimulus itself (e.g., certain sounds may innately elicit filial behavior in some species).
Nevertheless, the present study has demonstrated that functionally neutral aspects of a stimulus complex can acquire control over behavior that is initially elicited only by the complex as a whole. As such, the present findings resemble the observation of Pavlov (1927) that a dog's salivary reflex, normally elicited by food substances in contact with the mouth, is also evoked "when these substances are only those of smell and sight [p. 13]." The close parallel of these effects and those found in the present experiment is highlighted by Pavlov's conclusions that "... the effect of sight and smell of food is not due to an inborn reflex, but to a reflex which has been acquired in the course of the animal's own individual existence ... It is evident that the sight of food does not in itself act as a direct stimulus to salivary secretion. Only after the puppies have been allowed to eat bread and meat on several occasions does the sight or smell of these foodstuffs evoke the secretion [p. 22]."

Another set of findings that are very similar to those of the present experiment involves socialization in nonhuman primates. Infant monkeys display an immediate and apparently innate filial reaction (e.g., suppression of distress calling) to a mother surrogate which provides appropriate forms of tactile stimulation (Mason, Hill, \& Thompsen, 1971). In contrast, the ability of the surrogate to control distress calling when encased in plastic (and thus unavailable for contact) is acquired only after the infant has been raised with the claspable surrogate for several weeks. In this case, again, specific aspects of a stimulus complex which initially are functionally neutral appear to acquire behavioral control that the complex as a whole exerts from the beginning.

\section{REFERENCES}

Fischer, G. J. Auditory stimuli in imprinting. Journal of Comparative \& Physiological Psychology, 1966, 61, 271-273.

Gottlieb, G. Imprinting in relation to parental and species identification by avian neonates. Journal of Comparative \& Physiological Psychology, 1965, 59, 345-356.

Hoffman, H. S., Eiserer, L. A., \& Singer, D. Acquisition of behavioral control by a stationary imprinting stimulus. Psychonomic Science, 1972, 26, 146-148.

Hoffman, H. S, \& Ratner, A. M. A reinforcement model of imprinting: Implications for socialization in monkeys and men. Psychological Review, 1973,80,527-544.

Hoffman, H. S., Stratton, J. W., Newby, V., \& Barrett, J. E. Development of behavioral control by an imprinting stimulus. Journal of Comparative \& Physiological Psychology, 1970, 71, $229-236$.

Klopfer, P. H. An analysis of learning in young Anatidae.

Ecology, 1959, 40, 90-102.

Mason, W. A., Hill, S. D., \& Thompsen, C. E. Perceptual factors in the development of filial attachment. Proceedings of the 3rd International Primatological Congress, 1971, 3, 125-133.

Pavlov, I. P. Conditioned reflexes. (Trans. G. V. Anrep) London: Oxford University Press, 1927.

Sluckin, W. Imprinting and early learning. Chicago: Aldin 1965. 